Le Lait (1984), 64, 521-536

\title{
Eléments d'étude du procédé Bridel de fabrication de caséine à partir de lait décationisé par échanges d'ions (E.I.)
}

\author{
par
}

A. PIERRE et M. DOUIN

\author{
S u m m a r y \\ StUdies ON THE BRIDEL PROCESS \\ FOR INDUSTRIAL MANUFACTURE OF CASEIN FROM MILK ACIDIFIED \\ BY ION EXCHANGE
}

In a casein manufacture process recently developed by Bridel Society, one portion of skimmilk is acidified to $\mathrm{pH} 2.2$ by mixing with ion-exchange resin and added to another portion of non acidified skimmilk in order to obtain $p H 4.6$ in the mixture. Use of this process is claimed to lead to a casein yield increase of 3 to $5 \%$ in comparison to the classical acid process.

Study of the $p H$ variation kinetic allows to show that most of this yield increase comes from an insolubility hysteresis effect on proteosepeptone (P.P.) components. Rising the skimmilk pH from 2.2 to 4.6 maintains insoluble the component P.P. 5 which aggregates with caseins and so precipitates with them. In the classical acid casein process, $p H$ is lowered from 6.6 to 4.6 and that allows total solubility of P.P. 5 which is loosed in the whey. Retention of P.P. 5 in casein precipitate does not represent an adulteration of the product because it is known that this component is the 1-105 peptide resulting from plasmin action on $\beta$ casein in cow udder. The so observed hysteresis effect is likely due to amino-acid composition of this large peptide (5 phosphoserine and 14 glutamic acid residues).

Some retention of phospholipoproteins is also observed in the casein product obtained by the Bridel process by means of electrophoresis and thin layer chromatography. The lack of reliability of these analytical methods, as far as quantitation is concerned, does not sure evidence of another part of the observed casein yield increase.

Key words

Casein manufacture - Yield - Proteose-peptones.

I.N.R.A., Laboratoire de Recherches de Technologie Laitière, 65, rue de SaintBrieuc - 35042 Rennes (France).

Laiterie E. Bridel, B.P. 5 - 35240 Retiers. 


\section{R és u m é}

L'origine de l'augmentation du rendement en caséine découlant de l'emploi du procédé Bridel réside essentiellement dans la rétention de l'un des composants azotés du lactosérum, les protéoses peptones. Cette rétention est due à un effet d'hystérésis d'insolubilité lié au fait que le pH isoélectrique des caséines est atteint dans ce procédé en partant de valeurs de $\mathrm{pH}$ inférieures, au contraire des procédés classiques où l'ajustement du $\mathrm{pH}$ se fait par abaissement $\mathrm{du} \mathrm{pH}$ du lait de fabrication. La quantité de protéoses peptones retenue est d'environ $0,8 \mathrm{~g} / \mathrm{l}$ de lait, ce qui entraîne une augmentation du rendement en caséine de 3,3\%. Le suivi électrophorétique de la partition des composants de la fraction protéose-peptone entre caséine et lactosérum montre que c'est surtout le composant 5 (PP-5) qui est retenu dans la caséine issue de l'emploi du procédé Bridel. Etant donné que ce composé PP-5 est reconnu comme étant le fragment 1-105 découlant de l'action de la plasmine sur la caséine $\beta$, sa rétention dans les caséines industrielles ne peut être considérée comme une adultération. Il est probable que l'effet d'hystérésis constaté est lié à la composition en acides aminés (5 phosphosérines 14 résidus acide glutamique) de ce gros peptide (PM 12 000). La rétention dans la caséine des phospholipoprotéines (composant 3 des protéoses peptones) a été mise en évidence à la fois par électrophorèse et par chromatographie en couche mince, mais des problèmes analytiques ont empêché la détermination quantitative de cette fraction.

Mots clés

Caséine industrielle - Rendements - Protéoses-peptones.

\section{INTRODUCTION}

La Société Bridel a mis au point un procédé industriel de séparation de la caséine acide à partir de lait décationisé par échanges d'ions (E.I.), qui a fait l'objet d'un dépôt de brevet en 1980 (Rialland et Barbier). Il est actuellement utilisé dans l'usine de Lohéac (Ille-et-Vilaine) pour le traitement de 600000 litres de lait par jour. Dans ce procédé, une partie du lait écrémé est mise en contact avec une résine cationique sous forme $\mathrm{H}^{+}$, afin d'éliminer l'ensemble des cations du lait qui sont échangés pour des protons (tableau 1).

L'opération est réalisée à $10^{\circ} \mathrm{C}$ environ avec de la résine Dowex 88 dans un rapport de volume résine/lait de 0,1 . Le traitement entraîne une acidification du lait dont le $\mathrm{pH}$ est abaissé à une valeur de l'ordre de 2,2 à la sortie de la colonne de résine. $\mathrm{A}$ ce $\mathrm{pH}$, la caséine est à l'état soluble. Le lait décationisé est ensuite additionné de lait non traité ( $\mathrm{pH}$ 6,7) en proportion convenable (généralement proche de 1 pour 1) 
TABLEAU 1

Teneurs en cations du lait et du lait décationisé $(\mathrm{g} / \mathrm{kg})$

\begin{tabular}{l|c|c}
\hline & Lait & Lait décationisé \\
\cline { 2 - 3 } Calcium & 1,34 & 0,14 \\
Magnésium & 0,10 & 0,02 \\
Sodium & 0,44 & 0,12 \\
Potassium & 1,50 & 0,20 \\
\hline
\end{tabular}

pour obtenir dans le mélange un $\mathrm{pH}$ de l'ordre de 4,8 , considéré comme optimal pour la précipitation de la caséine. La température des produits est portée à $48^{\circ} \mathrm{C}$ environ dans la chambre de mélange, pour faciliter la précipitation.

Etant donné l'originalité du procédé par rapport à la fabrication traditionnelle de caséine par addition d'acide chlorhydrique au lait, une étude s'est avérée nécessaire pour déterminer le comportement des constituants du lait au cours des étapes technologiques. De plus, nous avons recherché l'origine de l'augmentation du rendement azoté en caséine observée au niveau industriel $(+3,8 \%)$. Nous avons étudié plus particulièrement la partition des fractions azotées du lait et du lait décationisé sous l'action de l'acidification.

\section{MATERIEL ET METHODES}

Les échantillons analysés étaient soit des produits industriels prélevés au cours d'un cycle de fabrication à l'usine, soit des échantillons obtenus au laboratoire grâce à un montage expérimental (figure 1) qui permettait de préparer du lait décationisé en continu, de manière à reproduire des conditions proches de celles utilisées à l'usine. La résine $(300 \mathrm{ml})$ était placée dans un récipient hermétique de 2 litres à trois tubulures. L'une servait à l'entrée du lait écrémé, l'autre à la sortie du lait décationisé ; la troisième recevait une électrode qui permettait de prendre le $\mathrm{pH}$ du milieu. Le débit d'entrée du lait était réglé par une pompe et maintenu à une valeur telle que le $\mathrm{pH}$ du mélange lait-résine ne soit pas supérieur à 2.2. La résine était maintenue en suspension dans le lait à l'aide d'un barreau aimanté actionné par un agitateur magnétique. Un volume de résine permettait de traiter 10 volumes de lait. La décationisation était faite sur du lait à $10^{\circ} \mathrm{C}$. 


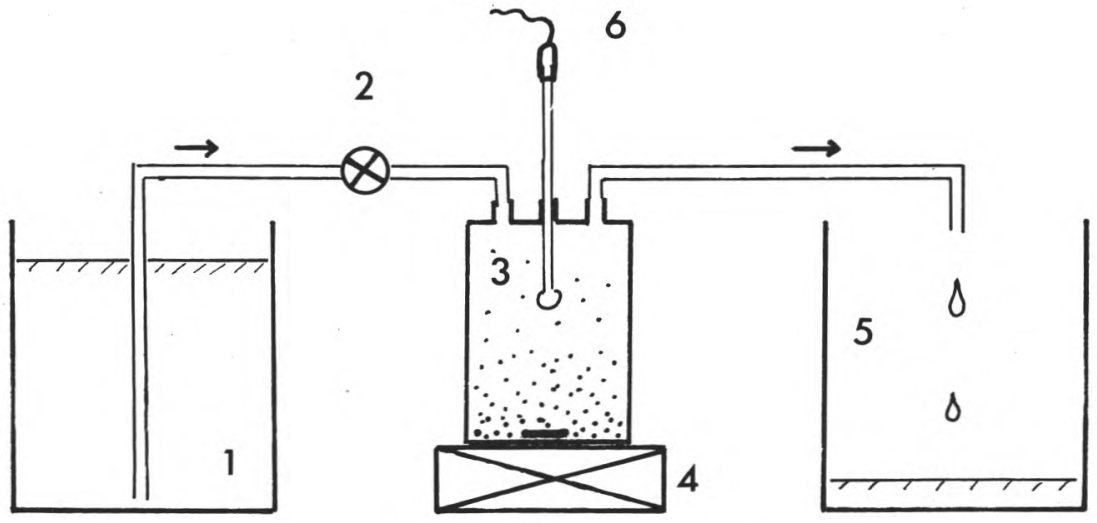

fig. 1

Schéma de l'appareil de décationisation utilisé au laboratoire

1. Réservoir de lait écrémé

2. Pompe d'alimentation

3. Chambre de contact résine-lait
4. Agitateur magnétique

5. Réservoir pour le lait décationisé

6. Electrode du pHmètre

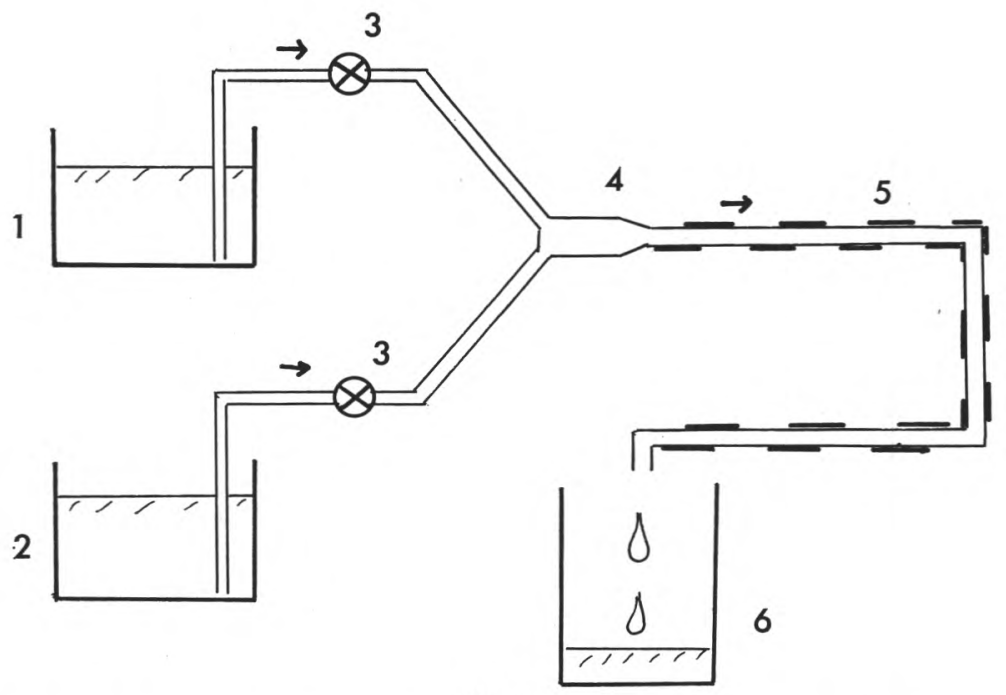

fig. 2

Schéma de l'appareil permettant de réaliser la coagulation de la caséine en continu au laboratoire

1. Réservoir de lait écrémé

2. Réservoir de lait décationisé ou d'acide chlorhydrique

3. Pompes d'alimentation

4. Chambre de mélange

5. Circuit de coagulation à $48^{\circ} \mathrm{C}$ avec ruban chauffant

6. Bac de réception du coagulum et du lactosérum 
La coagulation de la caséine acide était réalisée soit en continu dans une chambre de mélange (figure 2), soit en discontinu par acidification simultanée de tout le volume de lait à traiter. Grâce à ces montages, nous avons pu préparer de la caséine selon le protocole classique de fabrication par acidification chlorhydrique et selon le procédé E.I. Bridel.

La détermination de la matière azotée était réalisée à partir du dosage de l'azote selon la méthode de Kjeldahl en utilisant le coefficient $\mathrm{Nx}$ 6,38, même dans le cas de l'azote non protéique. Les fractions azotées non caséine (NCN) et non protéique (NPN) du lait étaient séparées selon la méthode de Aschaffenburg et Drewry (1959). Pour la préparation du NCN à partir du lait décationisé, la méthode a été adaptée au produit; l'addition d'acide acétique était supprimée et de l'acétate de sodium $\mathrm{N}$ était ajouté jusqu'à obtention d'un $\mathrm{pH}$ de 4,6 ; en fait, le volume d'acétate apporté était très peu différent de celui de la méthode originale. Les protéoses peptones de référence étaient isolées à partir d'un lait de mélange selon la technique de Andrews (1978). Pour l'extraction à partir des lactosérums, la durée du chauffage à $95^{\circ} \mathrm{C}$ était portée à 120 minutes et l'absence de protéines solubles non dénaturées dans le surnageant était vérifiée par HPLC selon la méthode décrite ci-après.

Le dosage des protéines solubles du lait, $\alpha$-lactalbumine et $\beta$-lactoglobuline, était réalisé par chromatographie d'exclusion HPLC (appareillage Varian - Colonne TSK $3000 \mathrm{SW}$ tampon d'élution TRIS 0,01 M pH 6,68; débit : $1 \mathrm{ml} / \mathrm{min}$ - détection à $280 \mathrm{~nm}$ ).

Les électrophorèses étaient effectuées selon la technique de Hillier (1976). Pour le dosage du composant 5 des protéoses peptones, la courbe d'étalonnage était établie à partir d'une solution de caséine $\beta$ purifiée ayant subi une protéolyse par la plasmine dans des conditions choisies pour que la totalité de la protéine soit hydrolysée, mais seulement au niveau de la liaison 105 ou 107 (caséine $\beta$ à $1 \%$ dans du

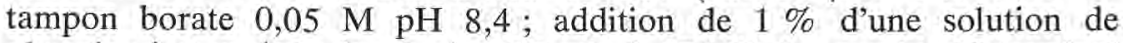
plasmine à $5 \mathrm{mg} / \mathrm{ml}$; incubation $90 \mathrm{~min}$ à $40^{\circ} \mathrm{C}$ ). Le peptide $\mathrm{N}$ terminal obtenu ( $M=12000$, selon Gordon et al., 1972) est le constituant 5 des protéoses peptones (Andrews, 1978). Dans les conditions d'hydrolyse totale, à une mole de caséine $\beta$ correspond une mole de composant 5 . Une hydrolyse plus prolongée conduirait à une perte de composant 5 (Andrews et Alichanidis, 1983).

Les mesures de turbidité ont été effectuées à $600 \mathrm{~nm}$.

Les phospholipides étaient extraits selon le procédé décrit par Bligh et Dyer (1959). La séparation était effectuée d'une part par chromatographie sur couche mince (Mangold et Kammereck, 1962), d'autre part en HPLC selon la méthode de Nissen et Kreysel (1983). L'extrait sec total était obtenu par passage à l'étuve $\left(105^{\circ} \mathrm{C}-7 \mathrm{~h}\right)$ des échantillons mélangés à du sable. Les minéraux étaient dosés par spectrophotométrie d'absorption atomique selon le protocole de Brulé et al. (1974). 


\section{RESULTATS}

La partition des protéines dans le lait et dans le lait décationisé sous l'effet d'un ajustement du pH à 4,6 a été étudiée selon plusieurs approches. Au laboratoire, nous avons d'une part dosé le NCN, d'autre part comparé les MAT des sérums séparés de ces laits par acidification à différents $\mathrm{pH}$, et celles des sérums obtenus lors de fabrications expérimentales. A l'usine, nous avons analysé la composition des sérums de fabrication.

\section{Dosage du NCN des laits}

Sur des laits normaux ou décationisés, nous avons effectué le dosage de la matière azotée totale et de la matière azotée non précipitable à $\mathrm{pH}$ 4,6 ( $\mathrm{NCN})$. Le tableau 2 rassemble les résultats obtenus sur 2 couples d'échantillons d'origine industrielle (laits décationisés ou non prélevés simultanément au cours d'un même cycle de fabrication) et sur 4 laits décationisés au laboratoire et analysés avant et après traitement. $\mathrm{La}$ teneur moyenne en MAT des laits est de $32,3 \mathrm{~g} / \mathrm{kg}$. Celle des laits décationisés de $30,3 \mathrm{~g} / \mathrm{kg}$. Cette différence de MAT est due pour une part à la rétention d'une fraction azotée non protéique du lait par la résine et pour une autre part à une dilution du lait par de l'eau interstitielle dans la colonne de résine. La perte en matière azotée non protéique sur la résine est d'environ $1 \%$ de la matière azotée totale, soit $0,3 \mathrm{~g} / 1$ en moyenne, et la dilution explique le reste de la différence, soit 1,7 g/l,

TABLEAU 2

Quantité de protéines du lait et du lait décationisé précipités à $\mathrm{pH} 4,6$ selon le protocole « $\mathrm{NCN} »(\mathrm{~N} \times 6,38 \mathrm{~g} / \mathrm{kg})$

\begin{tabular}{|c|c|c|c|c|c|c|c|}
\hline \multicolumn{2}{|c|}{$\begin{array}{l}\text { Origine } \\
\text { des } \\
\text { échantillons }\end{array}$} & \multirow{2}{*}{$\begin{array}{l}\text { MAT } \\
\\
34,7 \\
28,2 \\
33,4 \\
32,4\end{array}$} & \multicolumn{2}{|c|}{ 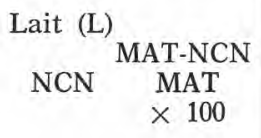 } & \multicolumn{3}{|c|}{ 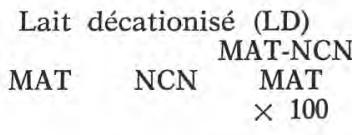 } \\
\hline Labo & $\begin{array}{l}1 \\
2 \\
3 \\
4\end{array}$ & & $\begin{array}{l}8,6 \\
6,5 \\
7,1 \\
7,5\end{array}$ & $\begin{array}{l}75,1 \\
79,7 \\
78,8 \\
76,9\end{array}$ & $\begin{array}{l}33,0 \\
27,1 \\
30,9 \\
30,2\end{array}$ & $\begin{array}{l}7,0 \\
5,4 \\
6,2 \\
5,6\end{array}$ & $\begin{array}{l}78,7 \\
80,1 \\
79,8 \\
81,5\end{array}$ \\
\hline Usine & $\begin{array}{l}5 \\
6 \\
7 \\
8\end{array}$ & $\begin{array}{l}33,4 \\
31,7\end{array}$ & $\begin{array}{l}7,2 \\
6,8\end{array}$ & $\begin{array}{l}78,4 \\
78,6\end{array}$ & 28,2 & 5,4 & 80,8 \\
\hline $\mathrm{n}=6$ & $\begin{array}{c}x \\
s \\
s / n\end{array}$ & $\begin{array}{r}32,3 \\
2,3 \\
0,9\end{array}$ & $\begin{array}{l}7,3 \\
0,7 \\
0,3\end{array}$ & $\begin{array}{r}77,9 \\
1,6 \\
0,6\end{array}$ & $\begin{array}{r}30,3 \\
2,3 \\
0,9\end{array}$ & $\begin{array}{l}5,9 \\
0,6 \\
0,3\end{array}$ & $\begin{array}{r}80,6 \\
1,3 \\
0,5\end{array}$ \\
\hline
\end{tabular}


c'est-à-dire $5,2 \%$. Les $\mathrm{NCN}$ sont respectivement de $7,3 \mathrm{~g} / \mathrm{kg}$ pour les laits et $5,9 \mathrm{~g} / \mathrm{kg}$ pour les laits décationisés. A partir de ces résultats, nous avons calculé (tableau 3) la quantité de matière azotée précipi-

\section{TABLEAU 3}

Interprétation de l'analyse du NCN des laits

\begin{tabular}{l|c|c}
\hline & $\begin{array}{c}\text { Lait } \\
\text { (L) }\end{array}$ & $\begin{array}{c}\text { Lait } \\
\text { décationisé } \\
\text { (LD) }\end{array}$ \\
\hline $\begin{array}{l}\text { Quantité de protéines précipitées MAT-NCN (g/kg) } \\
\begin{array}{l}\text { Dilution du LD (compte tenu de la perte de 1\% } \% \\
\text { en NPN sur la résine) : MATL - (MATLD + MATL) }\end{array}\end{array}$ & 25,0 & 24,4 \\
$\begin{array}{l}\text { Quantité théorique de protéines précipitables dans } \\
\text { le LD (MATL - NCNL) } \times(1-0,052)\end{array}$ & 0,052 \\
$\begin{array}{l}\text { Différence entre valeur expérimentale et théorique } \\
(\mathrm{g} / \mathrm{kg})\end{array}$ & 23,7 \\
\hline
\end{tabular}

table à $\mathrm{pH} 4,6$ obtenue pour les laits, $25,0 \mathrm{~g} / \mathrm{kg}$, et, pour les laits décationisés, $24,4 \mathrm{~g} / \mathrm{kg}$. Puis, nous avons déterminé par le calcul la quantité théorique de matière azotée précipitable à $\mathrm{pH}$ 4,6 du lait décationisé, compte tenu de la composition du lait normal et de la dilution observée, soit $23,7 \mathrm{~g} / \mathrm{kg}$. La différence entre la valeur expérimentale, $24,4 \mathrm{~g} / \mathrm{kg}$, et la valeur théorique, $23,7 \mathrm{~g} / \mathrm{kg}$, met en évidence une précipitation supplémentaire de $0,7 \mathrm{~g} / \mathrm{kg}$ de matière azotée dans le lait décationisé, ce qui représente une augmentation du rendement de précipitation de $3 \%$.

\section{Comparaison des lactosérums acides}

Nous avons préparé des lactosérums à partir de lait ou de lait décationisé dont le $\mathrm{pH}$ était amené à une valeur comprise entre 4,0 et 5,0. Le volume initial de l'échantillon de lait, $80 \mathrm{ml}$ environ, était porté après ajustement du $\mathrm{pH}$ à $100 \mathrm{ml}$, ce qui correspond à une dilution de 1,25. Nous avons choisi de travailler dans des conditions de faible dilution de manière à maintenir une force ionique élevée, et ainsi nous rapprocher des conditions de travail industrielles. Les lactosérums ont été séparés par filtration puis analysés pour leur teneur en MAT et en protéines solubles ( $\alpha$-lactalbumine et $\beta$-lactoglobuline). Leur composition en fonction du $\mathrm{pH}$ de séparation est reportée sur le graphique de la figure 3 . 


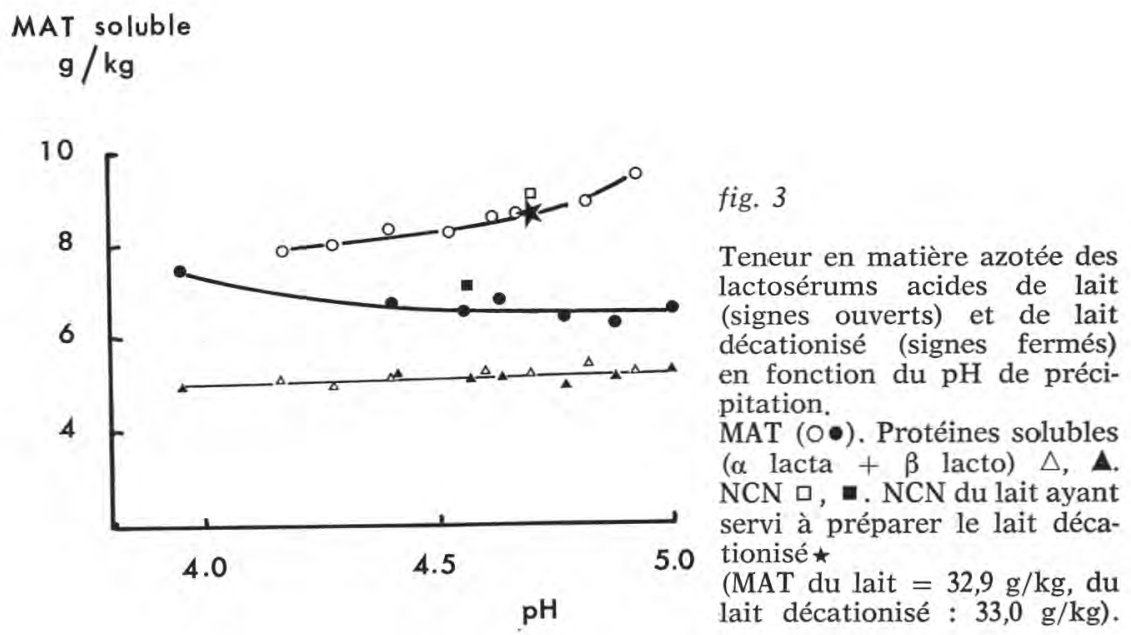

Les deux laits avaient une teneur en MAT très proche, $32,9 \mathrm{~g} / \mathrm{kg}$ pour le lait normal, $33 \mathrm{~g} / \mathrm{kg}$ pour le lait décationisé. La teneur en protéines solubles des lactosérums est identique pour les deux laits quel que soit le $\mathrm{pH}$. Par contre, la teneur en MAT des lactosérums du lait décationisé est toujours plus basse que celle du lait normal, $6,5 \mathrm{~g} / \mathrm{kg}$ à $\mathrm{pH} \mathrm{4,7} \mathrm{au}$ lieu de $8,5 \mathrm{~g} / \mathrm{kg}$ pour le lait normal.

On a isolé la fraction azotée soluble des laits selon la méthode NCN. La valeur obtenue est de $9 \mathrm{~g} / \mathrm{kg}$ pour le lait normal, $7 \mathrm{~g} / \mathrm{kg}$ pour le lait décationisé $(8,9 \mathrm{~g} / \mathrm{kg}$ pour le lait avant décationisation). Ces valeurs sont reportées sur la figure 3. Elles sont proches, mais un peu supérieures à celles des lactosérums au même $\mathrm{pH}$.

\section{Etude des lactosérums de fabrication de caséine}

Nous avons effectué au laboratoire des fabrications de caséine, selon la technologie E.I. Bridel ou par addition d'HCl, à partir du même lot de lait à teneur en NCN connue et nous avons analysé la composition en matière azotée totale des lactosérums obtenus. De même, des échantillons industriels de lait et lactosérum ont été prélevés à l'usine et analysés. Les résultats sont rassemblés dans le tableau 4.

Lorsque les deux types de fabrication sont effectués à partir du même lait, on observe que la teneur en MAT du sérum E.I. est plus faible que celle du sérum $\mathrm{HCl}$, de $1,5 \mathrm{~g} / \mathrm{kg}$ dans le premier essai, de $0,8 \mathrm{~g} / \mathrm{kg}$ dans le deuxième essai. La composition en MAT du lactosérum $\mathrm{HCl}$ est peu différente de celle du NCN du lait mis en œuvre. La MAT du lactosérum E.I. est intermédiaire entre la valeur du NCN du lait et celle du lait décationisé, pour les fabrications effectuées au laboratoire et pour les produits industriels. 


\section{TABLEAU 4}

Comparaison des fractions azotées des laits et de la teneur en MAT des lactosérums obtenus dans la fabrication des caséines $\mathrm{HCl}$ et E.I. $(\mathrm{N} \times 6,38-\mathrm{g} / \mathrm{kg})$

\begin{tabular}{|c|c|c|c|c|}
\hline \multicolumn{2}{|c|}{ Origine et nature des échantillons } & \multirow{2}{*}{$\begin{array}{r}\text { MAT } \\
33,4 \\
32,2 \\
6,2\end{array}$} & \multirow{2}{*}{$\begin{array}{c}\text { NCN } \\
7,2 \\
5,6 \\
6,2\end{array}$} & \multirow{2}{*}{$\begin{array}{r}\text { NPN } \\
1,7 \\
1,2 \\
1,5\end{array}$} \\
\hline Usine & $\begin{array}{l}\text { Lait } \\
\text { Lait décationisé } \\
\text { Lactosérum E.I. }\end{array}$ & & & \\
\hline Usine & $\begin{array}{l}\text { Lait } \\
\text { Lait décationisé } \\
\text { Lactosérum E.I. }\end{array}$ & $\begin{array}{r}31,7 \\
28,2 \\
5,6\end{array}$ & $\begin{array}{l}6,8 \\
5,4 \\
5,7\end{array}$ & $\begin{array}{l}1,5 \\
1,1 \\
1,2\end{array}$ \\
\hline Labo & $\begin{array}{l}\text { Lait } \\
\text { Lactosérum E.I. } \\
\text { Lactosérum HCl }\end{array}$ & $\begin{array}{r}32,1 \\
6,5 \\
8,0\end{array}$ & 7,8 & \\
\hline Labo & $\begin{array}{l}\text { Lait } \\
\text { Lait décationisé } \\
\text { Lactosérum E.I. } \\
\text { Lactosérum HCl }\end{array}$ & $\begin{array}{r}32,4 \\
32,4 \\
7,0 \\
7,8\end{array}$ & $\begin{array}{l}7,5 \\
6,0\end{array}$ & \\
\hline
\end{tabular}

\section{Suivi de la fraction protéose-peptone des laits}

Nous avons étudié la solubilité des protéoses peptones du lait dans les conditions de $\mathrm{pH}$ fixées lors de la fabrication de la caséine E.I.

Nous avons effectué une extraction quantitative de la fraction protéose peptone d'un lait de mélange, obtenant ainsi $4 \mathrm{~g}$ de protéines pour 4 litres de lait. Cet extrait nous a servi de mélange de référence dans la suite du travail.

Nous avons ensuite séparé la fraction protéose peptone de deux lactosérums acides $(\mathrm{pH} 4,6)$ préparés au laboratoire à partir de lait et de lait décationisé prélevés à l'usine ainsi que du lactosérum industriel E.I. correspondant. Les fractions isolées ont été soumises à une électrophorèse. La figure 4 permet de comparer les échantillons. Les quantités déposées sur le gel correspondent à des volumes identiques de lactosérum. Dans le mélange de référence apparaissent les trois constituants principaux des protéoses peptones, soit, par ordre de migration décroissante, les composants 8,5 et 3 . Le composant 5 représente $60 \%$ du total des protéoses peptones.

Les protéoses peptones du lactosérum de lait ont un profil peu différent de celles de la référence, par contre le lactosérum de lait acide et le lactosérum industriel E.I. sont appauvris en composant 5 ; pour le composant 3 on observe une moins grande différence. Le dosage du composant 5 donne une concentration de $1 \mathrm{~g} / \mathrm{kg}$ dans le lait, $0,6 \mathrm{~g} / \mathrm{kg}$ 


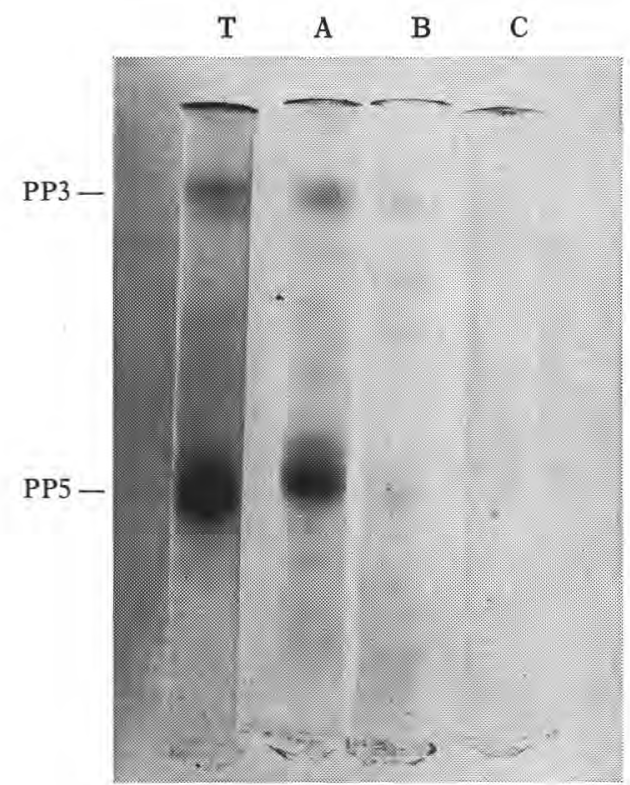

fig. 4

Mise en évidence des protéoses peptones dans les sérums acides de lait (A), de lait décationisé (B) et dans un sérum industriel de fabrication E.I. (C). Protéoses peptones de référence $(T)$.

dans le sérum de lait, $0,2 \mathrm{~g} / \mathrm{kg}$ dans le sérum de lait décationisé et $0,1 \mathrm{~g} / \mathrm{kg}$ dans le sérum industriel E.I. Le suivi de trois fabrications nous a donné les mêmes résultats. La précision du dosage est de $15 \%$.

La solubilité des protéoses peptones est dépendante du $\mathrm{pH}$. Sur la figure 5 , nous avons reporté la densité optique à $600 \mathrm{~nm}$ (D.O.) d'une solution de protéoses peptones dans de l'ultrafiltrat $(20 \mathrm{mg} / \mathrm{ml}, \mathrm{pH} 7)$ en fonction du $\mathrm{pH}$. Le $\mathrm{pH}$ est graduellement abaissé de $\mathrm{pH} 7$ à $\mathrm{pH} 2$ par addition d' $\mathrm{HCl} 3 \mathrm{~N}$ en ménageant un temps de repos de 4 minutes à chaque $\mathrm{pH}$ faisant l'objet d'une mesure de D.O. La D.O. initiale est de 0,650 ; elle reste stable jusqu'au $\mathrm{pH} 4,2$ au delà duquel se produit une brutale augmentation, qui s'accentue et se maintient aux $\mathrm{pH}$ plus bas. Le $\mathrm{pH}$ de la solution est alors relevé par addition de soude $2 \mathrm{~N}$;

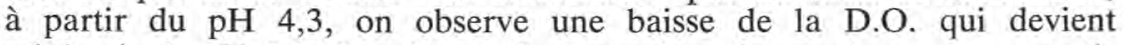
minimale à $\mathrm{pH} 5,2$. La vitesse de formation des agrégats au cours de l'acidification est rapide, elle se fait en moins d'une minute à $\mathrm{pH} 4,2$. Par contre leur dissociation au cours de l'augmentation du $\mathrm{pH}$ est plus

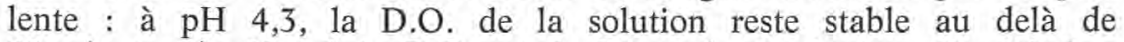
90 minutes, à $\mathrm{pH} 4,6$, la D.O. est de 2,3 après 4 minutes, elle s'abaisse à 0,650 après 90 minutes; à $\mathrm{pH} 4,9$ la D.O. diminue très rapidement dans les 4 premières minutes pour se fixer à la valeur de 1,8 . Plus le $\mathrm{pH}$ est élevé, plus la vitesse de dissociation est grande. A pH 5,1 elle est instantanée.

La dilution totale entrânée par l'addition d'acide, puis de soude lorsque le $\mathrm{pH}$ atteignait 7,0, était de 1,08 . 
D.O.

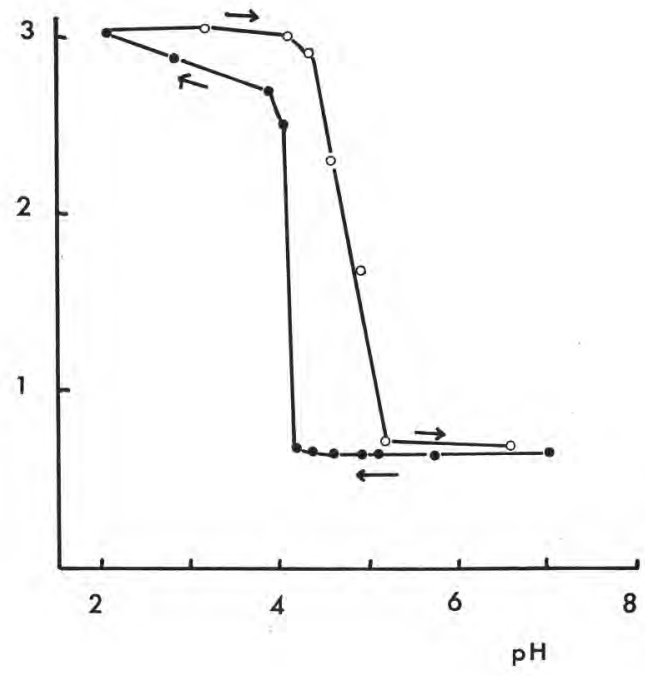

fig. 5

Evolution de la turbidité d'une solution de protéoses peptones dans l'ultrafiltrat en fonction du $\mathrm{pH}$ $(20 \mathrm{mg} / \mathrm{ml})$. D.O. $600 \mathrm{~nm}$.

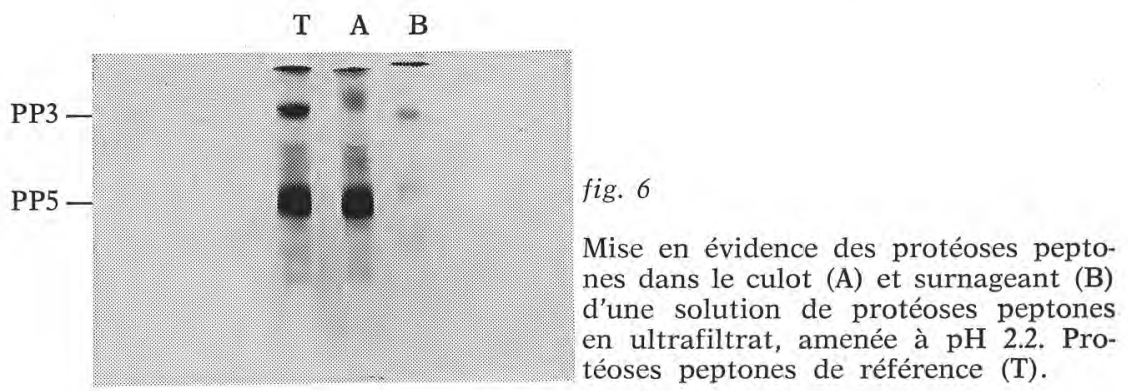

Une expérience identique a été réalisée à $40^{\circ} \mathrm{C}$; les courbes d'association et de dissociation en fonction du $\mathrm{pH}$ semblent peu modifiées par la température.

La solution précédente a été ramenée à $\mathrm{pH} 2,2$, puis centrifugée. Le culot de centrifugation a été redissous dans un volume de tampon tris $\mathrm{pH} 8,1$ égal au volume initial de l'ultrafiltrat. Le surnageant et le culot ont été analysés par électrophorèse (figure 6). Le culot contient la plus grande partie des protéoses peptones. Le surnageant contient encore un peu de composant 3.

\section{Suivi des protéines solubles}

Nous avons préparé au laboratoire de la caséine selon le procédé E.I. Bridel et selon le procédé $\mathrm{HCl}$, à partir du même lait. La teneur 
TABLEAU 5

Teneur en protéines solubles des lactosérums des deux types de fabrication de caséine $(\mathrm{g} / \mathrm{l})$

\begin{tabular}{|c|c|c|c|c|c|c|c|c|}
\hline \multicolumn{2}{|c|}{$\begin{array}{l}\text { Origine } \\
\text { de } \\
\text { l'échantillon }\end{array}$} & \multirow[t]{2}{*}{$\begin{array}{l}\text { Lait } \\
\text { (1) }\end{array}$} & \multirow[t]{2}{*}{$\begin{array}{c}\text { Lactosérum } \\
\mathrm{HCl} \\
\text { (2) }\end{array}$} & \multicolumn{2}{|c|}{$\begin{array}{l}\text { Différence } \\
\text { lait-sérum } \\
\text { (1) - (2) }\end{array}$} & \multirow[t]{2}{*}{$\begin{array}{l}\text { Lactosérum } \\
\text { E.I. } \\
\text { (3) }\end{array}$} & \multicolumn{2}{|c|}{$\begin{array}{l}\text { Différence } \\
\text { lait-sérum } \\
\text { (1) - (3) }\end{array}$} \\
\hline \multicolumn{6}{|l|}{ Labo } & & & \\
\hline Essai 1 & $\begin{array}{l}\beta \text { lacto } \\
\alpha \text { lacta }\end{array}$ & $\begin{array}{l}4,3 \\
1,2\end{array}$ & $\begin{array}{l}3,5 \\
0,9\end{array}$ & $\begin{array}{l}0,8 \\
0,3\end{array}$ & 1,1 & $\begin{array}{l}3,5 \\
0,9\end{array}$ & $\begin{array}{l}0,8 \\
0,3\end{array}$ & 1,1 \\
\hline Essai 2 & $\begin{array}{l}\beta \text { lacto } \\
\alpha \text { lacta }\end{array}$ & $\begin{array}{l}4,8 \\
1,4\end{array}$ & $\begin{array}{l}4,3 \\
1,3\end{array}$ & $\begin{array}{l}0,5 \\
0,1\end{array}$ & 0,6 & $\begin{array}{l}4,4 \\
1,4\end{array}$ & 0,4 & 0,4 \\
\hline Essai 3 & $\begin{array}{l}\beta \text { lacto } \\
\alpha \text { lacta }\end{array}$ & $\begin{array}{l}4,6 \\
1,2\end{array}$ & $\begin{array}{l}4,2 \\
1,3\end{array}$ & 0,4 & 0,4 & $\begin{array}{l}4,2 \\
1,2\end{array}$ & 0,4 & 0,4 \\
\hline \multicolumn{9}{|c|}{ Usine $\mathrm{n}=14$} \\
\hline & $\begin{array}{r}\beta \text { lacto } x \\
\mathrm{~s}\end{array}$ & $\begin{array}{l}4,41 \\
0,28\end{array}$ & & & & $\begin{array}{l}3,56 \\
0,20\end{array}$ & 0,85 & 1,09 \\
\hline & $\alpha \begin{array}{r}\alpha \text { lacta } x \\
s\end{array}$ & $\begin{array}{l}1,15 \\
0,11\end{array}$ & & & & $\begin{array}{l}0,91 \\
0,11\end{array}$ & 0,24 & \\
\hline
\end{tabular}

en $\alpha$-lactalbumine et en $\beta$-lactoglobuline des laits et des lactosérums a été déterminée. Les résultats obtenus lors de trois essais différents sont rassemblés sur le tableau 5 . On remarque que, pour un même essai, le lactosérum $\mathrm{HCl}$ et le lactosérum E.I. ont la même teneur en $\alpha$-lactalbumine et $\beta$-lactoglobuline. Le lait et le lactosérum industriels. E.I. ont été analysés également lors de 14 prélèvements effectués à l'usine au cours d'une semaine. La composition moyenne des laits industriels est assez proche de celle du lait de l'essai $\mathrm{n}^{\circ} 1$ du laboratoire. La composition moyenne des lactosérums industriels est également très proche de celle du lactosérum E.I. de l'essai $\mathrm{n}^{\circ} 1 \mathrm{du}$ laboratoire.

\section{Suivi des phospholipides}

Des phospholipides sont associés aux lipoprotéines du lait et nous les avons étudiés en tant que traceur pour mettre en évidence la présence de ces lipoprotéines. Celles-ci sont extraites au cours de l'isolement des protéoses peptones, dont elles constituent le composant 3.

Nous avons effectué un suivi des phospholipides dans les produits séparés au cours de la fabrication du caséinate. La méthode d'extraction utilisée s'est avérée peu reproductible. Nous avons cependant pu mettre en évidence la présence des phospholipides dans le lait et dans le lait décationisé. Les lactosérums $\mathrm{HCl}$ et E.I. obtenus au laboratoire par la 
méthode en continu présentent peu de différence dans leur teneur en phospholipides; au contraire, les lactosérums obtenus par la méthode discontinue sont très différents : le lactosérum $\mathrm{HCl}$ est riche en phospholipides tandis que le lactosérum E.I. en est dépourvu, ce qui lui confère une grande limpidité.

Des phospholipides sont présents dans les caséines préparées selon les deux types de technologies, soit industriellement, soit au laboratoire.

\section{CONCLUSION}

Nous avons montré que la solubilité des protéoses peptones du lait dépend du $\mathrm{pH}$; le composant 5 est insoluble à un $\mathrm{pH}$ inférieur à 4,2 et le composant 3 partiellement précipité. La dispersion des associations protéiques formées se produit au $\mathrm{pH}$ de 4,9 . Les $\mathrm{pH}$ d'agrégation $(4,2)$ et de dispersion $(4,9)$ sont donc légèrement différents. Cette observation peut être expliquée en partie par la cinétique du processus qui serait rapide dans le sens de la formation des agrégats et plus lente dans le sens de leur dissociation. Il est possible qu'il existe pour le composant 5 deux $\mathrm{pH}$ de moindre stabilité. En effet, sa séquence (fraction $\mathrm{N}_{\mathrm{t}}$ de la caséine $\beta$-résidus 1 à 105 ou 107) est caractérisée par la présence de 5 résidus phosphosérine et de 14 résidus acide glutamique qui lui confèrent une forte charge négative. Le $\mathrm{pH}$ de la fonction acide secondaire de l'acide glutamique est de l'ordre de 4,25; celui des phosphosérines, pour ce peptide en solution dans de l'ultrafiltrat, pourrait être de l'ordre de 4,9. Nous pensons donc que la solubilisation du peptide requiert l'ionisation des deux types de fonctions et, inversement, l'agrégation ne se produit que lorsqu'elles sont toutes neutralisées.

Cette propriété peut expliquer les observations qui ont été faites sur les produits industriels. Dans le lait, les protéoses peptones sont à l'état soluble, par contre, dans le lait décationisé, elles ont tendance à s'agréger, en raison du $\mathrm{pH}(2,2)$; il est probable qu'elles s'adsorbent sur la caséine et soient ainsi stabilisées. Lorsqu'on effectue le fractionnement de la matière azotée des laits selon le protocole du $\mathrm{NCN}$, le $\mathrm{pH}$ est amené de 6,7 à 4,7 dans le cas du lait et les protéoses peptones restent en solution puisque le $\mathrm{pH}$ critique au cours de l'acidification est de 4,2. Dans le cas du lait décationisé, le pH est remonté de 2,2 à 4,7; les protéoses peptones restent adsorbées sur la caséine et précipitent avec elle ; il faudrait atteindre le $\mathrm{pH}$ de 4,9 pour les solubiliser. La différence de valeur des NCN, observée dans le tableau 2 pour le lait et le lait décationisé (compte tenu de la dilution) est donc expliquée par la différence de solubilité des protéoses peptones dans les conditions expérimentales adoptées.

Dans la fabrication industrielle de caséine, selon le procédé E.I. la coagulation est provoquée par le mélange du lait normal et du lait décationisé en quantités souhaitables pour obtenir un $\mathrm{pH}$ de 4,8 (ce qui 
correspond approximativement à un mélange volume à volume). L'augmentation du $\mathrm{pH}$ du lait décationisé est insuffisante pour solubiliser ses protéoses peptones et l'abaissement de $\mathrm{pH}$ du lait normal trop faible pour insolubiliser celles présentes dans cette fraction du mélange : théoriquement, seules les protéoses peptones (fraction 5) provenant du lait acide devraient être entraînées dans le coagulum. En fait, nous observons que le lactosérum de fabrication industrielle ne contient pratiquement plus de composant 5 , et peu de composant 3 . Les conditions industrielles sont donc telles que ces composants sont largement retenus sur la caséine. Il est probable que la cinétique des gradients de $\mathrm{pH}$, de température, d'agitation et de force ionique du mélange lait normal + lait acide réalisée dans la chambre de mélange est différente de celle réalisée au laboratoire. La valeur critique du $\mathrm{pH}$ d'agrégation des protéoses peptones du lait normal s'en trouve modifiée.

Lors de la fabrication de caséine selon le procédé E.I. Bridel, la plus grande partie du composant 5 des protéoses peptones des laits mis en œuvre est incorporée à la caséine; le composant 5 étant une fraction de la caséine $\beta$ (Andrews, 1978), ceci ne constitue nullement une adultération du produit. Le composant 3 est aussi partiellement précipité, ce qui entraîne une clarification du lactosérum. Cependant, le comportement de cette fraction de lipoprotéines semble assez variable selon les essais; la précipitation semble totale dans les essais en discontinu et partielle dans les essais en continu.

L'extraction des protéoses peptones à partir d'un lait nous a donné un rendement de $1 \mathrm{~g}$ par litre. Andrews (1978) en avait obtenu 1,28 g. Il est probable que cette teneur soit assez variable d'un lot de lait à l'autre. Si l'ensemble de cette fraction était retenu lors de la fabrication de caséine, cela entraînerait une augmentation maximum du rendement en protéines de 4 à $5 \%$ (calculé sur la base de $25 \mathrm{~g}$ de caséine par $\mathrm{kg}$ de lait et $1 \mathrm{~g}$ à $1,28 \mathrm{~g}$ de protéoses peptones). Le composant 5 représente seulement une partie de cette fraction, $60 \%$ d'après nos résultats. Sa teneur dans les laits serait donc comprise entre $0,6 \mathrm{~g}$ et $0,78 \mathrm{~g} / \mathrm{kg}$, soit un gain de rendement théorique en caséine de 2,4 à $3,2 \%$ dû à cette fraction seulement.

Les résultats expérimentaux obtenus par dosage direct du composant 5 dans les produits industriels E.I. (lait et lactosérum industriel) permettent de calculer un gain réel en protéines de 3,6\% dû à cette fraction. La comparaison avec une fabrication industrielle de type $\mathrm{HCl}$ n'a pu être effectuée ; mais, d'après la composition d'un lait normal et de son lactosérum $\mathrm{HCl}$ préparé au laboratoire, il apparaît que le composant 5 est beaucoup plus faiblement retenu et représente seulement $1,6 \%$ de la caséine $\mathrm{HCl}$. Cette valeur est difficilement extrapolable et devrait être confirmée par l'analyse de produits industriels. Entre les deux types de fabrication, l'écart de rendement dû à la différence de rétention du composant 5 est donc de $2 \%$. Cette fraction représente $60 \%$ des protéoses peptones, si l'on admet que le composant 3 est retenu dans les mêmes proportions, on peut calculer un gain de rende- 
ment total de $2 \times 100 / 60=3,3 \%$, soit $0,8 \mathrm{~g} / \mathrm{kg}$ de lait. Toutefois les déterminations quantitatives obtenues à partir des électrophorèses restent approximatives, car la méthode a une faible précision. D'autre part, nous n'avons travaillé que sur 3 cycles industriels.

L'étude du fractionnement de la matière azotée des laits (tableaux 2 et 3) avait permis de conclure à la précipitation de $0,7 \mathrm{~g} / \mathrm{kg}$ de protéines supplémentaires dans le lait décationisé. Cette valeur correspond bien, comme ordre de grandeur, avec la fraction protéose peptone sensible au $\mathrm{pH}$ que nous avons mise en évidence.

Les résultats de l'étude sur les teneurs en protéines solubles des lactosérums ne semblent pas faire apparaître de différence entre la technologie $\mathrm{HCl}$ et E.I. Cependant, une certaine variabilité est observée d'un essai à l'autre. Elle doit résulter de différences dans les traitements technologiques appliqués aux laits. Pour ce qui est de l'étude des phospholipides, elle a été entravée par l'utilisation d'une méthode d'extraction non fiable pour les produits liquides.

En conclusion de ces travaux, il semble que l'augmentation du rendement en caséine observée en utilisant le procédé E.I. Bridel puisse être imputée à l'incorporation d'une partie des protéoses peptones dans la caséine.

\section{Remerciements}

Nous remercion J.L. Maubois, J.P. Barbier, G. Brulé, J.P. Rialland et J.P. Gallacier pour l'intérêt qu'ils ont porté au déroulement de notre travail et pour d'utiles discussions.

\section{Bibliographie}

ANDREWS (A.T.) (1978). - The composition, structure and origin of proteosepeptone Component 5 of bovine milk. European Journal of Biochemistry, 90, 59-65.

Andrews (A.T.) and Altchandis (E.) (1983). - Proteolysis of casein and the proteose-peptone fraction of bovine milk. Journal of Dairy Research, 50, 275-290.

AschafFenbuRg (R.) and DREWRY (J.) (1959). - New procedure for the routine determination of the various non casein proteins of milk. International Dairy Congress, vol. 3, 5-a-59, 1631-1637.

Bligh (E.G.) and Dyer (W.J.) (1959), - A rapid method of total lipid extraction and purification. Canadian Journal of Biochemistry and Physiology, 37, 8 911-917.

Brulê (G.), Maubois (J.L.) et Fauquant (J.) (1974). - Etude de la teneur en éléments minéraux des produits obtenus lors de l'ultrafiltration du lait sur membrane. Le Lait, 54, 600-615.

Gordon (W.G.), Groves (M.L.), Greenberg (R.), Jones (S.B.), Kalan (E.B.), PeterSON (R.F.) and TownENd (R.E.) (1972). - Probable identification of -, T.S. $\mathrm{R}$ - and S - caseins as fragments of casein. Journal of Dairy Science, 55, 261-263. 
HILLIER (R.M.) (1976). - The quantitative measurement of whey proteins using P.A.E. Journal of Dairy Research, 43 (2), 259-265.

MANGold (H.K.) and KammerecK (R.) (1962). - New methods of Analysing industrial aliphatic lipids. Journal of American Oil Chemists Society, 39, 201-206.

Nissen (H.P.) and KREYSEL (H.W.) (1983). - Analysis of phospholipids in human semen by H.P.L.C. Journal of Chromatography, 276, 29-35.

Rialland (J.P.), et BARBIER (J.P.) (1980). - Brevet français $\mathrm{n}^{\circ}$ de publication 2480568. Procédé de traitement du lait par une résine échangeuse de cations en vue de la fabrication de la caséine et du lactosérum. 